\title{
Properties of dynamical fractal geometries in the model of causal dynamical triangulations
}

\author{
J. Ambjorn $\oplus^{1, *}$ Z. Drogosz $\oplus^{2, \dagger}$ A. Görlich $\oplus^{2, \ddagger}$ and J. Jurkiewicz $\oplus^{2, \S}$ \\ ${ }^{1}$ The Niels Bohr Institute, Copenhagen University, Blegdamsvej 17, DK-2100 Copenhagen Ø, Denmark \\ and IMAPP, Radboud University, Nijmegen, PO Box 9010, the Netherlands \\ ${ }^{2}$ Institute of Theoretical Physics, Jagiellonian University, Lojasiewicza 11, Kraków PL 30-348, Poland
}

(Received 28 January 2021; accepted 30 March 2021; published 27 April 2021)

\begin{abstract}
We investigate the geometry of a quantum universe with the topology of the four-torus. The study of noncontractible geodesic loops reveals that a typical quantum geometry consists of a small semiclassical toroidal bulk part, dressed with many outgrowths, which contain most of the four-volume and which have almost spherical topologies, but nevertheless are quite fractal.
\end{abstract}

DOI: 10.1103/PhysRevD.103.086022

\section{INTRODUCTION}

Causal dynamical triangulation (CDT) is a model that attempts to apply methods of quantum field theory in the context of a quantum model of geometric degrees of freedom. ${ }^{1}$ As will be described below, the model comes with a (proper) time, whereas the description of the geometries in the spatial directions is genuinely coordinate independent. The existence of the time coordinate has been instrumental for the construction of an effective minisuperspace action of the quantum theory, where we have integrated over the spatial geometries. In particular, it allowed us to talk about the emergence of a semiclassical minisuperspace geometry, as well as quantum fluctuations thereof [15-19]. There is no reason not to expect a similar emergence of geometry in the spatial directions. However, bearing in mind the importance of the proper time coordinate in our analysis of the minisuperspace geometry, it might be preferable to reintroduce some aspects of coordinates in the spatial directions, too. In some sense this is

\footnotetext{
*ambjorn@nbi.dk

tzbigniew.drogosz@doctoral.uj.edu.pl

*andrzej.goerlich@uj.edu.pl

§erzy.jurkiewicz@uj.edu.pl

${ }^{1}$ Reviews of the model can be found in $[1,2]$. The main idea is to have a lattice model of quantum gravity where one in a nonperturbative way can test the idea of asymptotic safety [3-8]. Models of dynamical triangulations (DT) were earlier attempts in this direction [9-11], which however did not work, but see [12-14] for recent attempts to revive that class of lattice models.

Published by the American Physical Society under the terms of the Creative Commons Attribution 4.0 International license. Further distribution of this work must maintain attribution to the author(s) and the published article's title, journal citation, and DOI. Funded by SCOAP ${ }^{3}$.
}

against the spirit of general relativity which is coordinate independent, but coordinates can be very useful.

In our recent paper [20] we discussed one possibility of reintroducing coordinates in CDT in the spatial directions. In many of the former studies of four-dimensional CDT, the spatial topology was chosen to be that of a three-sphere $S^{3}$, and, given a spatial geometry as it appears in the path integral, we know of no simple way of reintroducing useful spatial coordinates in that case. However, in [20] the spatial topology of the Universe was chosen to be that of a threetorus $T^{3}$. A $d$-dimensional manifold with a toroidal topology can be viewed as consisting of an elementary cell, which is periodically repeated infinitely many times in all $d$ directions. Although the choice of the elementary cell is not unique, the possibility of introducing such an object enables the use of its boundaries as a reference frame, with respect to which a Cartesian-like system of coordinates determined by the geodesic distance to the boundaries may be constructed. One conclusion drawn from the analysis in [20] was that the geometry of a typical triangulation which appears in the CDT path integral is surprisingly fractal. Before trying to extract any emergent spatial geometry from such triangulations it is thus important to understand the spatial geometry of a typical quantum configuration better. For that purpose we have found it advantageous to use topological observables: closed noncontractible geodesic loops, connecting the same geometric object in different copies of the elementary cell. The distribution of the length of shortest loops with a given set of winding numbers passing through particular elements of geometry yields information about geometric structures, and this kind of analysis has been used successfully in the study of two-dimensional Euclidean quantum gravity [21].

The upper part of Fig. 1 provides a two-dimensional illustration of what we are looking for in the case of the higher dimensional tori. We imagine that we have an 


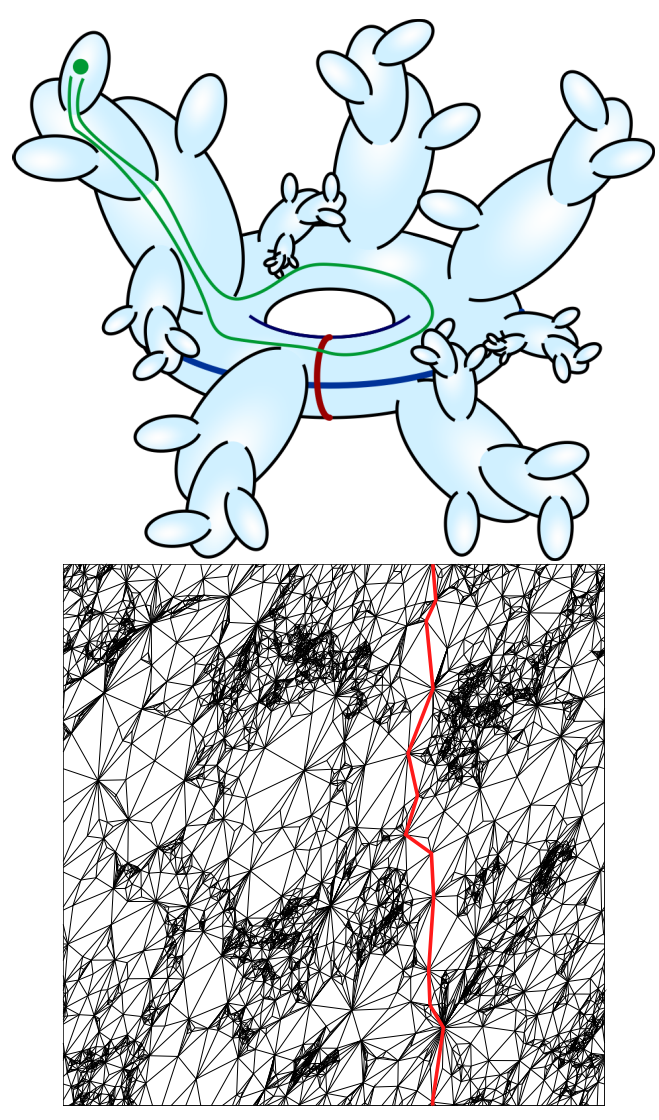

FIG. 1. Up: Illustration of a torus with outgrowths. The blue and red lines represent two nonequivalent and noncontractible loops. The green loop is the shortest loop passing through the green point in the same direction as the blue line. Down: Embedding of a triangulation of the two-torus consisting of 150000 triangles into the Euclidean plane (picture from [21]). Shown in red is the shortest noncontractible loop.

underlying "semiclassical" toroidal structure, but there can be many outgrowths, which can be viewed as its quantum fluctuations. A point in an outgrowth will have a long noncontractible geodesic loop passing through it, while a point on the "semiclassical" toroidal part will have a short noncontractible geodesic loop. In this way one can map out the geometry of the toroidal universe in considerable detail, as will be described below. An example point in an outgrowth is marked with a green dot in the upper part of Fig. 1. and the green line is a noncontractible geodesic loop for this point.

In the lower part of Fig. 1 we have shown how a twodimensional toroidal quantum configuration looks. The configuration is a two-dimensional triangulation made of 150000 equilateral triangles, generated by Monte Carlo simulations of two-dimensional Euclidean quantum gravity. By a conformal mapping the triangulation can be mapped to an elementary cell in the plane. What is shown is a piecewise-linear approximation to this mapping (plus an affine mapping to make it a square). The figure illustrates how such a quantum configuration consists of mountains (outgrowths) and valleys. By far the most twovolume (the greatest number of triangles) is contained in the outgrowths, as can readily be seen from the picture. In four-dimensional CDT, we consider paths that connect centers of simplices, i.e., which consist of edges of the dual triangulation (see Sec. II). In the two-dimensional case, the red line shown in the lower part of Fig. 1 consists of links of the direct lattice. The picture shows quite precisely the fractal structure of two-dimensional quantum gravity. It is known that the Hausdorff dimension of spacetime in twodimensional quantum gravity is four and not two, as one might perhaps naively expect [22-24]. On a regular torus consisting of $N$ triangles one would expect a shortest loop of length approximately $N^{1 / 2}$ links. However, here we see that the length is much closer to $N^{1 / 4}$. In particular, this implies that the number of triangles in the valleys scale as $N^{1 / 2}$, and not proportionally to $N$. The area of the valleys will thus disappear in an $N \rightarrow \infty$ limit where the continuum area $V \propto N a^{2}$ is kept fixed, $a$ being the length of a link in the triangulation before it was projected onto the plane. Therefore, in the two-dimensional case the valleys are not semiclassical, but a quantum phenomenon. We expect the situation to be different in the case of a four-dimensional CDT torus, the reason being that the Hausdorff dimension of a typical CDT configuration is four, i.e., the same as the canonical dimension of the spacetime. We might then have a picture where the valleys of $T^{3}$ constitute a semiclassical configuration which can act as a starting point for a description of a semiclassical spatial geometry. This is one of the points we will investigate in this article.

The rest of the article is organized as follows: in Sec. II we shortly define the CDT model of quantum gravity, in order to fix the notation (we refer to [1,2] for more detailed definitions). In Sec. III we define certain characteristics which are special for spacetimes with toroidal topologies. Section IV describes how the Monte Carlo simulations are performed, whereas Sec. V reports on the measurements of the shortest loops of winding number one. In Sec. VI these measurements are generalized to loops with higher winding numbers. In Sec. VII we generalize even the possibilities of higher winding numbers, acknowledging the fact that our winding numbers are dependent on our chosen reference frame and that a true geometric winding can be any linear combination of our labeling of windings. Section VIII discusses if simplices in the outgrowths and simplices in the valleys have different geometric neighborhoods, possibly signifying that the valleys can be viewed as semiclassical, while the outgrowths might be viewed as quantum fluctuations. Finally, Sec. IX contains a discussion of the results and our conclusions.

\section{THE MODEL}

The basic idea in CDT is to calculate the quantum amplitude of the transition between two physical 
states. The amplitude is defined as a path integral over field configurations, which in this case are spacetime geometries,

$$
\mathcal{Z}=\int \mathcal{D}\left[g_{\mu \nu}\right] e^{i S_{\mathrm{EH}}\left[g_{\mu \nu}\right]}
$$

$S_{\mathrm{EH}}$ is the Einstein-Hilbert action

$$
S_{\mathrm{EH}}\left[g_{\mu \nu}\right]=\frac{1}{16 \pi G} \int_{M} d^{4} x \sqrt{-\operatorname{det} g}(R-2 \Lambda),
$$

where $R$ is the scalar curvature and $\Lambda$ is the cosmological constant. This expression is formal and requires regularization and a precise definition of both the integration measure over $g_{\mu \nu}$ and the domain of integration over spacetimes. In CDT it is assumed that we will take into account only spacetimes that admit a global time foliation: $M=\Sigma \times I$. The term causality in the context of the model means that the topology of space $\Sigma$ is preserved in time evolution. An additional assumption is that the spatial topology of the Universe is closed. Corresponding to $I$ there is an initial and a final global time for the geometries considered, and the amplitude (1) is the transition amplitude between the spatial geometries at the initial and final global times. This amplitude can be calculated analytically if spacetime is two-dimensional [25], but in the case of three- or four-dimensional spacetime we have to rely on numerical simulations, and thus a discretization of spacetime geometries.

The spacetime geometries are discretized using a method based on an idea of Regge [26], and the diffeomorphisminvariant integral over metrics (1) is regularized by a sum over a set of simplicial manifolds with a correct topological structure. For each spacetime of this kind it is possible to perform Wick rotation to Euclidean signature, after which the exponent in the sum becomes real and the complex amplitudes become real probabilities (see [27] for details):

$$
\mathcal{P}(\mathcal{T}) \propto e^{-S(\mathcal{T})} .
$$

This formulation is well suited to numerical simulations, which, as mentioned, are the main tool used in the analysis. The foliation of spacetime defines an ordering on the slices (leaves) $\Sigma$, each of which can in a natural way be assigned an integer time parameter $t$.

In the $3+1$-dimensional case, the spacetime is built out of four-dimensional simplices. Each of them is the convex hull of five vertices that lie on two neighboring slices $\Sigma$. There are thus two types of four-simplices: $\{4,1\}$-simplices with four vertices on a slice $t$ and one vertex on a slice $t \pm 1$, and $\{3,2\}$-simplices with three vertices on a slice $t$ and two vertices on a slice $t \pm 1$. Each simplex abuts along its threedimensional faces on five other simplices, called its neighbors. All spacelike links, i.e., line segments which connect two vertices on the same time slice, are of length $a_{s}$, and all timelike links, i.e., line segments which connect two vertices on neighboring time slices, are of length $a_{t}$. Those lengths are unchanging, and their ratio squared is the asymmetry factor: $\alpha=a_{t}^{2} / a_{s}^{2}$.

The Regge action (the Hilbert-Einstein action on a piecewise linear manifold) for a causal triangulation depends only on global quantities:

$$
\begin{aligned}
S_{\mathrm{EH}}(\mathcal{T})= & -\left(K_{0}+6 \Delta\right) N_{0}+K_{4}\left(N^{\{4,1\}}+N^{\{3,2\}}\right) \\
& +\Delta \cdot N^{\{4,1\}},
\end{aligned}
$$

where $N_{0}, N^{\{4,1\}}$ and $N^{\{3,2\}}$ denote the total number of vertices and of $\{4,1\}$ - and $\{3,2\}$-simplices in the configuration. The three dimensionless coupling constants, $K_{0}$, $K_{4}$ and $\Delta$, are related, respectively, to the inverse of the gravitational constant $G^{-1}$, the cosmological constant $\Lambda$, and the asymmetry factor $\alpha$.

To describe a configuration fully, one has to do the following:

(i) choose the initial and final states. To avoid the problem of making such a choice, we customarily adopt the periodic boundary conditions with some number of time slices $T$;

(ii) label all the vertices and all the four-simplices;

(iii) list all the vertex labels together with corresponding time parameters;

(iv) list all the four-simplex labels together with the quintuples of their vertices and their neighbors placed opposite to the vertices.

The same data are contained in the dual description, which is a graph (called the dual lattice) whose vertices correspond to the four-simplices of the configuration, and whose links correspond to interfaces between the four-simplices.

As mentioned, no analytic solution for the model exists in $3+1$ dimensions. Therefore, we probe the trajectory space by random generation of configurations with desired topology and scrutinize the results. The configurations are not created one-by-one from scratch, but instead they are generated in large number by performing a Monte Carlo simulation, which starts from a very simple triangulation and lets it gradually evolve by means of 7 types of geometric moves. The moves modify the configuration locally in a topology-preserving way and are ergodic, which means that by performing them it is possible to obtain any triangulation with the same topology. In every simulation many billions of moves are performed, which allows us to overcome autocorrelation and to generate independent configurations. Moves are performed at random in a way satisfying the detailed balance condition and with correct probabilities derived from the action. We set the values of the couplings $K_{0}$ and $\Delta$ before starting the simulation in order to study the model at a chosen point in the coupling constant space (cf. [28]). The number of triangulations grows exponentially with 
$N_{4}=N^{\{4,1\}}+N^{\{3,2\}}$ for a fixed topology. ${ }^{2}$ Summing over all triangulations with a fixed $N_{4}$, using as a weight $e^{-S_{\mathrm{EH}}(\mathcal{T})}$ for each triangulation $\mathcal{T}$, will result (to leading order in $N_{4}$ ) in an expression

$$
\mathcal{Z}_{N_{4}}\left(K_{0}, \Delta, K_{4}\right) \propto e^{\left(K_{4}^{\text {crit }}\left(K_{0}, \Delta\right)-K_{4}\right) N_{4}},
$$

and the full discretized version of (1) is then

$$
\mathcal{Z}\left(K_{0}, \Delta, K_{4}\right)=\sum_{N_{4}} \mathcal{Z}_{N_{4}}\left(K_{0}, \Delta, K_{4}\right) .
$$

In general we are interested in the limit where the average value of $N_{4} \rightarrow \infty$, which corresponds to the limit $K_{4} \rightarrow K_{4}^{\text {crit }}{ }^{+}$. In simulations, taking this limit is replaced by studying the properties of a sequence of spacetimes, each with a fixed $N_{4}$ and $N_{4} \rightarrow \infty$.

\section{TOPOLOGY, BOUNDARIES AND COORDINATES}

In the original formulation of CDT in four dimensions it was assumed that the spatial topology of time slices was spherical $\left(S^{3}\right)$. For technical reasons, related to the computer simulations, it was assumed that time was periodic. However, this periodicity played no role in the initial study of universes with $S^{3}$ topology as long as the time period was sufficiently large. The existence of the time foliation sufficed to analyze the phase structure of the model. The phase diagram is surprisingly complex when one considers the extreme simplicity of the action (4), which only depends on the global quantities $N_{0}, N^{\{4,1\}}$ and $N^{\{3,1\}}$. Of the four different phases, only one, the so-called $C$ phase (also called the de Sitter phase) seems to be related in a simple way to semiclassical spacetimes, and we will here only discuss results obtained when the coupling constants are chosen such that the system is in this phase. The simplest observable measured was the spatial volume profile $N_{3}(t)$, defined as the number of spatial tetrahedra on a time slice $t$. A typical system with a sufficiently large number of slices $T$ consisted of a blob and a cutoff size stalk (necessary to satisfy the periodic time boundary conditions mentioned above). Owing to the invariance with respect to (discrete) translations in time, the position of the blob could be arbitrarily shifted in time. We used this possibility to center it around a fixed time position. It was shown that both the average volume $\left\langle N_{3}(t)\right\rangle$ and its fluctuations can be derived from the discretized version of the effective minisuperspace action [30] for the isotropically homogeneous 4D universe. The classical solution in this case corresponded to a 4D de Sitter sphere. It should be noted that although the minisuperspace model was

\footnotetext{
${ }^{2}$ There is no analytical proof of this, only numerical evidence [29].
}

originally proposed in the context of $4 \mathrm{D}$ general relativity [31], where all degrees of freedom except the scale factor were suppressed, the situation in CDT is different. The first difference is that $N_{3}(t)$ corresponds to a collective state, where all degrees of freedom are integrated out. The second difference is the sign of the effective action, opposite to the one found in general relativity. In CDT the solution of classical equations of motion gives a stable classical vacuum state, where at each $t$ we have all possible geometric realizations with a particular value of $N_{3}(t)$. The existence of this highly nontrivial classical general relativity limit of the model was one of the most important results in the early studies of CDT. It also raises the question about precisely what continuum theory CDT will describe (if any). As we mentioned above, a time foliation is built into the CDT formulation. From this point of view, the CDT setup seems to be the natural one if one wants to study Hořava-Lifshitz gravity [32,33], and such studies have indeed been conducted in the case of a three-dimensional spacetime [34]. In four dimensions, Hořava-Lifshitz gravity contains higher derivative terms, which makes the theory perturbatively renormalizable. Such terms are not explicitly introduced into our CDT action. Effectively, however, the parameter $\Delta$ appears as a new coupling constant, and it could play the same role as higher derivative terms in a continuum limit. Thus, our model could well be describing Hořava-Lifshitz gravity rather than general relativity (GR), as has also been advocated in [35]. Support for this can be found in the case of twodimensional CDT, which can be solved analytically, and where it was shown that it corresponds to a quantized version of Hořava-Lifshitz gravity [36]. Finally, from a Wilsonian point of view, one would expect that a continuum limit of CDT leading to Hořava-Lifshitz gravity is more likely than a GR-limit, which has an enhanced symmetry between space and time.

It is thus an interesting question to what extent the semiclassical limit can be broadened to include degrees of freedom in spatial directions, and it in turn might resolve the question of whether four-dimensional CDT should be viewed as a lattice version of GR or Hořava-Lifshitz gravity. The simplicity of the $S^{3}$ topology, however, makes such analysis very difficult or even impossible to perform because of the background independence. We do not have any reference system with respect to which observables could be measured. This may be different if we decide to formulate the model with a richer spatial topology. In the analysis presented in this article we chose $\Sigma=T^{3}$, and for technical reasons (ease of computer implementation and eschewal of the need for selecting the initial and the final states) we imposed periodic boundary conditions in the time direction: $\mathcal{M}=T^{3} \times T^{1}$.

Thus, each configuration is topologically a Cartesian product of four circles. Each closed curve within the configuration is homotopically equivalent to a combination 
of those circles, and the coefficients of that linear combination are the four winding numbers of the loop. Let us call them the winding numbers in the $x, y, z$ and $t$ directions.

One can equivalently consider the four-torus as an infinite periodic system. All the $N_{4}$ simplices of the torus are contained in an elementary cell, which repeats itself infinitely many times in four directions. The elementary cell can be defined in various ways, each of which is equivalent to a choice of a set of faces between neighboring four-simplices to form the cell's four boundaries. A loop within the torus corresponds in this picture to a path joining the same simplices in two different copies of the elementary cell. We can assign a set of four numbers to each copy of the elementary cell in such a way that the differences between them for any two copies are identical to the four winding numbers of the corresponding loop.

Arguably it is the most convenient to look at loops in the dual picture, and so henceforth we will most often use the word "loop" to mean not a spacetime curve but an ordered set of connected simplices whose image in the dual lattice is a noncontractible directed cycle. The length of a loop is the number of links in the cycle. (For simplicity we assume that all links have the same length.) Similarly, a geodesic between two simplices will mean a line connecting them whose image in the dual lattice has minimal length.

The distance (the minimal number of links in the dual lattice) from a simplex to each of the boundaries of the elementary cell serves as pseudo-Cartesian coordinates of the simplex. This definition was studied in a previous paper [20]. In a regular hypercubic lattice a sum of distances from any simplex to the two opposite boundaries is a constant, equal to the geodesic distance between the boundaries. On a random lattice generated by CDT this is, however, not the case. We observe a nontrivial distribution of these values (see Fig. 2), which may indicate either that the shape of the elementary cell is far from being rectangular, or that quantum fluctuations of the geometry can be viewed as "mountains" and in effect simplices close to the top of the mountains have a larger geodesic distance to the boundaries than those lying in the valleys between the mountains. Results indicate that both effects may be important. The latter effect is supported by the difference visible in Fig. 2 between the distributions $P\left(x+x^{\prime}\right)$ for all simplices (solid lines) and simplices adjacent to a boundary (dotted line, $x=1$ or $x^{\prime}=1$ ). Boundaries are chosen to locally minimize their area, thus they prefer the central region of a torus (valleys) and omit outgrowths (mountains). Therefore, simplices adjacent to one boundary are closer to the second boundary than an average simplex.

In this paper we will try to perform a closer analysis of relevant structures produced in simulations to understand the properties of the quantum landscape. Of a primary interest will be correlations between valleys, which in this picture can be interpreted as a semiclassical background geometry.
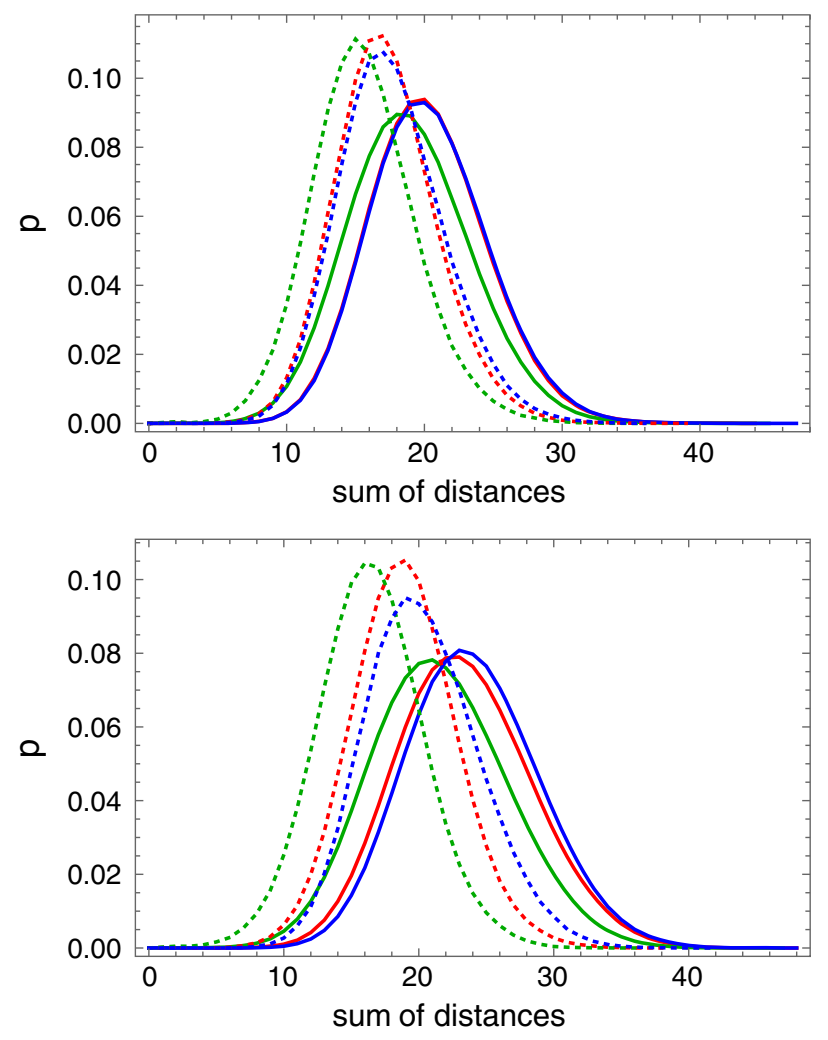

FIG. 2. Distributions of a sum of distances from simplices to the two opposite boundaries in the $x$ direction (red), the $y$ direction (green) and $z$ direction (blue) for systems with $N^{\{4,1\}}=80 \mathrm{k}$ (up) and $N^{\{4,1\}}=160 \mathrm{k}$ (down). The distributions scale consistently with the Hausdorff dimension $d_{H}=4$. The dotted lines refer to simplices adjacent to the boundary, $x=1$ or $x^{\prime}=1$, respectively, for the two sides of the boundary.

\section{DESCRIPTION OF SIMULATIONS}

The starting point of all the Monte Carlo simulations was a single simple configuration, which contained 4096 simplices in $4^{4}$ regularly placed four-dimensional hypercubes. The considered configuration consisted of $T=4$ time slices. Interfaces between some neighboring simplices were chosen as the boundaries of the elementary cell (cf. Fig. 3). The precise shape of the initial configuration and the initial position of the boundaries can be chosen freely as long as they have the correct topology.

The boundaries are encoded as an additional set of numbers assigned to every dual link in a triangulation. Each link $\{i j\}$ in a dual lattice is characterized by a set of four numbers $n_{i j}^{\mu}= \pm 1,0$, where nonzero values mean that the link crosses the corresponding boundary in a positive or negative direction. Here, $\mu=1, \ldots, 4$ enumerates directions. These numbers have an obvious property $n_{i j}^{\mu}=-n_{j i}^{\mu}$, and their sums along a closed loop reproduce the winding numbers of the loop.

In order to keep the size of the boundary small, after each performed move a procedure that changes the position of 


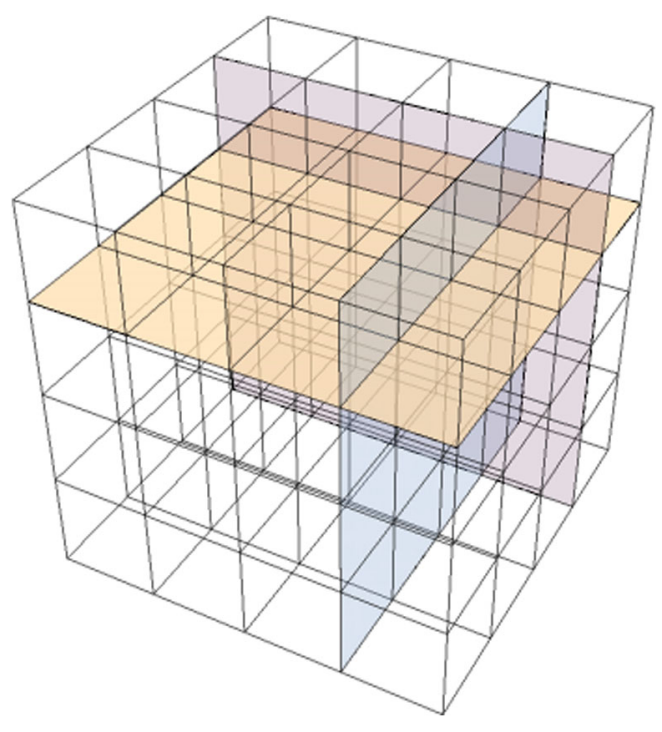

FIG. 3. A schematic view of a single time slice in the initial configuration. Visible are the $4^{3}$ hypercubes (each of which is divided into 16 simplices) and the starting position of the boundaries.

boundary if more than two faces of a single simplex belong to it was invoked in the region affected by the move.

The simulations were performed at the canonical point in the phase space of toroidal CDT, i.e., in the $C$ phase, for the parameters $K_{0}=2.2$ and $\Delta=0.6$ and for $N^{\{4,1\}}=160000$. For the analysis we chose a typical, well-thermalized configuration. The total number of simplices of the configuration we analyzed was equal to $N_{4}=N^{\{4,1\}}+N^{\{3,2\}}=370724$.

\section{SHORTEST LOOPS}

In a previous article [20] we introduced the idea of analyzing the shortest loops of nonzero winding numbers passing through a given simplex to gain understanding of the shape of the system. We described the distribution of lengths of loops with low winding numbers and noted the universality of its shape (cf. Fig. 4). We also noted the strong correlations of distribution of loop lengths in different directions.

To recapitulate, in order to find the shortest loop of a given winding number passing through a simplex, we treat the four-torus as an infinite periodic system and follow step by step the front of a diffusion wave beginning at the chosen simplex (using a diffusion wave in a system infinite in four directions is applicable to the case of low winding numbers; otherwise this method becomes computationally inefficient and should be modified, cf. Sec. VI). The number of loops with a given winding number that pass through a simplex grows (eventually) exponentially fast with the loop length. Thus, while it is feasible to list all the shortest loops of a given type, in the case of longer loops we
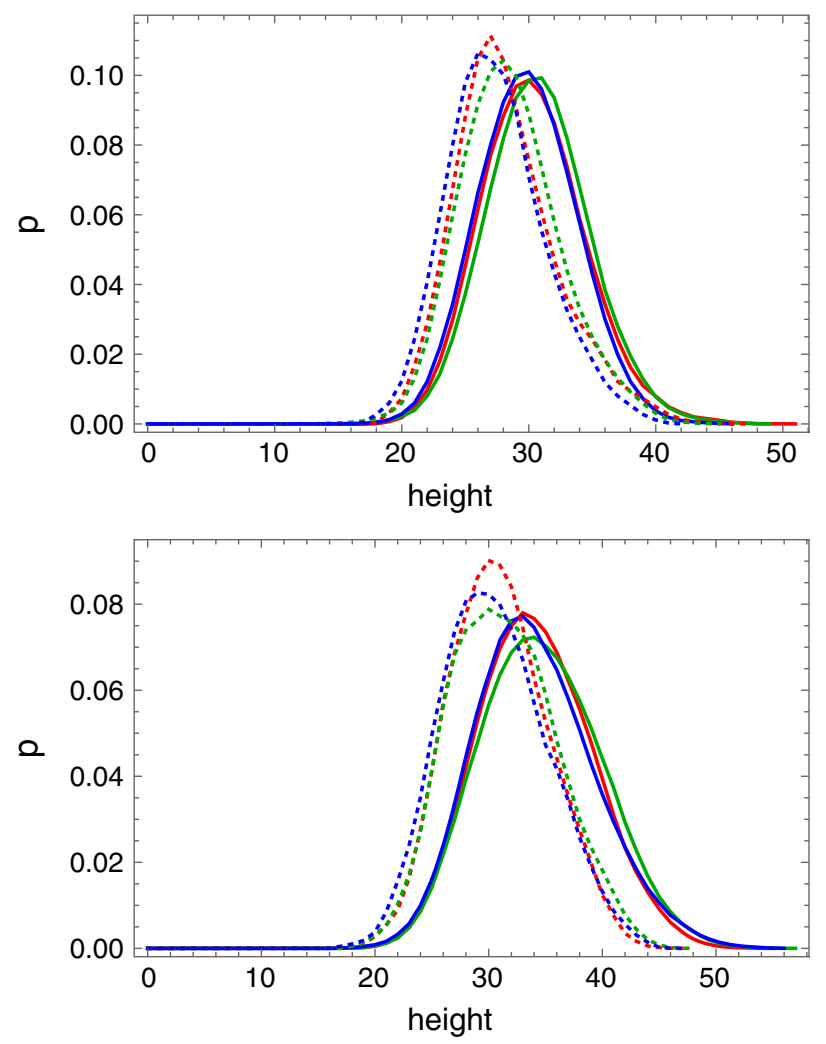

FIG. 4. Distributions of distances from simplices to their copies in neighboring elementary cells (heights) for systems with $N^{\{4,1\}}=80 \mathrm{k}$ (up) and $N^{\{4,1\}}=160 \mathrm{k}$ (down). These are lengths of minimal loops with winding numbers $\{1,0,0,0\}$ (red), $\{0,1,0,0\}$ (green) and $\{0,0,1,0\}$ (blue) shifted in $r$ by a shift of order one. The dotted lines refer to simplices adjacent to the boundary.

usually have to pick one sample loop, representing their universal properties.

Figure 5 presents the connections between simplices forming the shortest loops of winding numbers $\{1,0,0,0\}$ in the configuration. It is evident that such very short loops are rare: in a configuration containing $N_{4}=370724$ simplices there are only 20 simplices belonging to loops of length 18. Moreover, loops of length from 19 to 21 often differ from each other only by a few simplices; the number of separate short loops of the same length-the number of distinct deepest valleys - is very small. The results in the other spatial directions are similar.

Geometry of the random manifold generated by computer simulation is highly fractal. It is tempting to interpret the distribution of the lengths of loops with a unit winding number in spatial or time directions, having its maximum at a length above 30 , as a signal that in most cases the starting simplex is located inside one of the fractal structures (mountains, outgrowths), whereas the (rarer) simplices belonging to the loops with a length that is minimal or close to minimal correspond to the (relatively simple) basic structure of valleys in the configuration. With this 


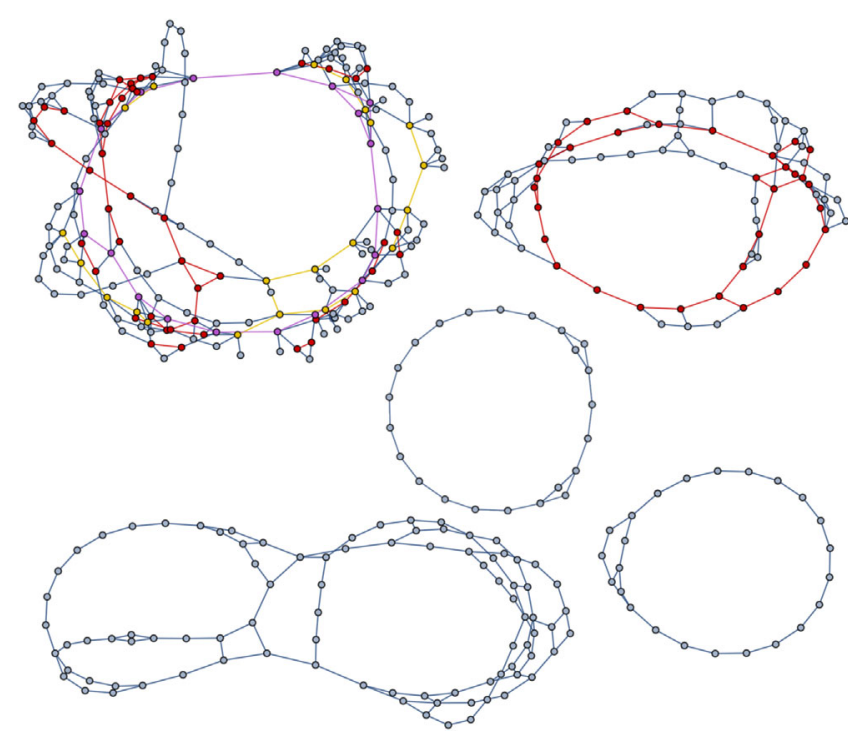

FIG. 5. The dual-lattice graphs of all the connections between simplices of lowest x-heights: 18 (purple), 19 (red), 20 (yellow) and 21 (blue). In general, loops longer than the minimal length almost always contain fragments belonging to shorter loops, but for small heights there exist also a small number of loops built only of simplices with equal x-height.

interpretation, the length of a loop starting from a particular simplex reflects the position of the simplex relative to the valleys. And so, for a particular configuration we assign to each simplex a unique set of four numbers: lengths of loops with a unit winding number in a particular direction and zero in the other three directions. Following these numbers along simplices belonging to any particular loop, we may see how far the simplices belonging to the loop are from the base, and how this distance changes along the loop. The four numbers assigned to a simplex can be called its heights, as they reflect its position above the basic structure. For the sake of brevity, we can use the names $x$-height, $y$-height, z-height, t-height for the length of loops in the unit directions. It was checked that, as expected, the height values of the five neighbors of any simplex differ from its own height by $\pm 2, \pm 1$ or 0 . In general any loop starting deep inside a fractal structure is expected to move closer to the base and then eventually climb back to simplices in the same fractal. There are only few loops whose simplices are all of equal height. This property is possessed by the shortest loop in the configuration and a few dozen other short loops, which are, so to speak, the "locally deepest valleys". To summarize, the height of a simplex is defined as the length of the shortest loop passing through it. It is determined separately for each topological dimension of the torus. For a given simplex and direction, there might exist, and often do, several shortest loops, all with the length equal to the height of the simplex. In further analysis we pick only one of them for each simplex. Usually, through each simplex pass also many loops (of the same length or longer) that are minimal for other simplices (see the discussion near the end of Sec. VII).

\section{LOOPS WITH HIGHER WINDING NUMBERS}

The short loops contain important information about the underlying structure of the manifold and about the distribution of valleys. The choice of four directions $(x, y, z$ and $t$ ) is nevertheless to a large extent arbitrary. It reflects a particular structure of the initial configuration and the memory about the initially chosen elementary cell. It may give a false impression that the elementary cell remains geometrically hypercubic during the thermalization. We can extend the analysis of minimal loop length distributions to include simplices in cells with an arbitrary set of winding numbers $\left\{n^{\mu}\right\}$. The analysis shows that the network of minimal loops contains not only loops in the four basic directions, as discussed above, but also loops with nontrivial winding numbers. It is important to note, that the winding numbers of a loop do not depend on a particular choice of boundaries or, equivalently, the elementary cell.

Using a four-dimensional diffusion wave in a system treated as infinite in four directions is a simple method to ensure that we find the shortest loop of a given winding number, regardless of the shape of the dual lattice, as the diffusion wave cannot "miss" any short path. One could continue the diffusion to find loops with any higher winding numbers. However, the number of visited simplices at a distance $R$ from the initial simplex grows as $R^{3}$, which means that eventually, for large $R$, the procedure would become too time- and memory-consuming, and computationally inefficient. We should, therefore, modify the boundary conditions in such a way that the number of simplices visited by the diffusion wave grows more slowly. One example is to consider the four-torus as a system infinite in only one direction-for example the $\mathrm{x}$-direction-and strictly periodic in all other directions. This way we can measure loops with winding numbers $\{n, 0,0,0\}, n=1,2, \ldots$. A small modification of this idea is to assume that cell boundaries in all directions except $x$ are impenetrable for the diffusion wave. In both of the methods the number of visited simplices in the $R^{\text {th }}$ shell, for $R$ large enough, stabilizes and becomes independent of the distance $R$. We will observe a faster growth only up to the range where the diffusion wave reaches the boundaries of the system in the finite directions. A similar result can be obtained if we put the impenetrable walls in all directions except $x$ at boundaries between cells number \pm 1 and \pm 2 . This choice improves the behavior of the diffusion process in case the initial simplex is near the boundary of the elementary cell.

In Fig. 6 we present results of using this method (with impenetrable walls in all directions except $x$ at boundaries between cells number \pm 1 and \pm 2 ) to measure the average lengths of loops with winding numbers $\{n, 0,0,0\}$, 


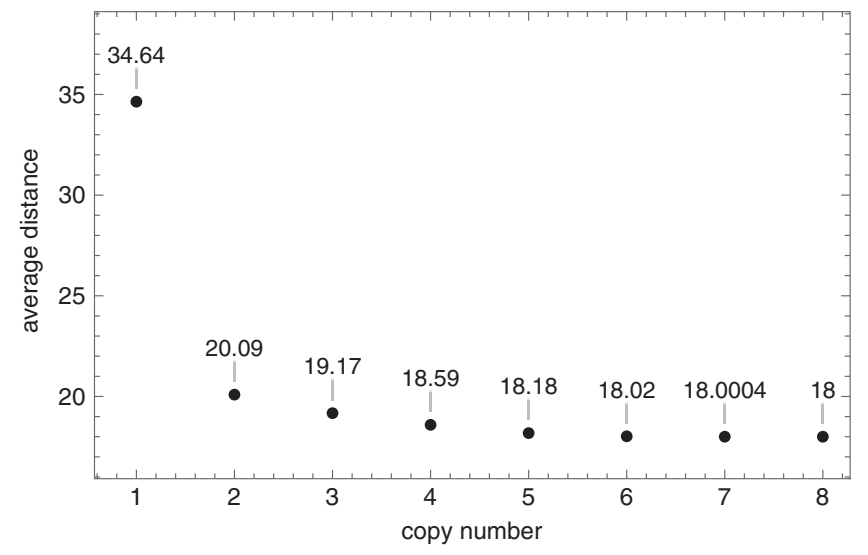

FIG. 6. Distance from a starting simplex to its copy in cell $\{n, 0,0,0\}$ minus distance from the same simplex to its copy in cell $\{n-1,0,0,0\}$, averaged over all the simplices of the configuration. Already for $n=8$ the minimal value of 18 is reached.

$n=1,2, \ldots, 8$. Similar measurements were performed in yand z-directions. It turns out that the difference between the distance to the copy number $n$ and the distance to the copy number $n-1$ of a given simplex decreases rapidly with increasing $n$, down to the length of the minimal shortest loop in the given direction (lowest height of a simplex), and then it remains constant. The explanation is simple: in order to minimize the length of a loop with a high winding number $n$ in a given direction, it becomes advantageous for the loop to connect the initial simplex to a simplex of the lowest possible height, then trace the shortest loop of unit winding number $n$ times, and finally return to the initial simplex. As can be seen in Fig. 6, already for $n=8$ the shortest loop of the configuration is a part of all the loops of winding number $n$. The figure shows the distances averaged over all simplices in the configuration. We note that the average for loops with a unit winding number in the $\mathrm{x}$ direction for this particular configuration is 34.64 , which is well above the minimal length of 18 , showing that most simplices belong to fractal structures.

Figure 7 shows the heights of consecutive simplices along loops with a growing sequence of winding numbers in the $\mathrm{x}$-direction, starting from a simplex lying far within such a fractal structure, called also an outgrowth. We can see that as the winding number of the loop increases, usually the minimal $\mathrm{x}$-height of the simplices belonging to the loop decreases, ultimately down to 18 , which is the length of the shortest $\{1,0,0,0\}$ loops in the configuration. As there are no two completely separate $\mathrm{x}$-loops of length 18 , this means that all the loops of a high winding number pass many times mostly through the same set of simplices. The graphs showing the heights in the $\mathrm{y}, \mathrm{z}$ and $\mathrm{t}$ directions for the same set of loops demonstrate that although there is a correlation between height in all the directions, the correlation is not perfect, especially after the loop leaves
TABLE I. Lengths of the shortest loops in the three basic spatial directions.

\begin{tabular}{rrrrr}
\hline \hline \multicolumn{4}{c}{ Winding numbers } & \\
\hline $\mathrm{x}$ & $\mathrm{y}$ & $\mathrm{z}$ & $\mathrm{t}$ & Length \\
\hline 1 & 0 & 0 & 0 & 18 \\
1 & -1 & 1 & 0 & 16 \\
2 & -1 & 1 & 0 & 27 \\
0 & 1 & 0 & 0 & 19 \\
0 & 1 & 0 & 1 & 16 \\
-1 & 1 & -1 & 0 & 16 \\
-2 & 3 & -1 & 1 & 43 \\
1 & 0 & 0 & 0 & 18 \\
1 & -1 & 1 & 0 & 16 \\
2 & -1 & 3 & 0 & 47 \\
\hline \hline
\end{tabular}

the outgrowth. The repeating sawlike pattern is a loop of a high winding number tracing one of the shortest loops of unit winding number several times. The heights of simplices belonging to a loop that is shortest in the $\mathrm{x}$-direction are not minimal in the other three directions. However, even though in the other directions the height fluctuates, it still remains close to the minimal height, as the simplices in the semiclassical region have low heights in all directions.

\section{ALTERNATIVE BOUNDARY CONDITIONS}

The other method, mentioned in Sec. VI, is searching for a shortest path connecting a simplex to its copy in another elementary cell in a system that is infinite in one direction and has periodic boundary directions imposed in the other three directions. The values of the winding numbers in the other directions are irrelevant for this method, i.e., we find loops with winding numbers of the form $\{n, a, b, c\}, a, b, c$ being any integers, instead of only $\{n, 0,0,0\}$.

It turns out that also for these boundary conditions paths starting at various simplices tend to converge and follow a handful of very short loops. However, those loops are almost never the shortest loops with unit winding numbers, e.g., $\{1,0,0,0\}$. Rather than that, those loops have winding numbers of the form $\{n, a, b, c\}$ yet are shorter than $\mathrm{n}$ times the length of the shortest $\{1,0,0,0\}$ loop in the configuration (see Table I; we used data from a diffusion in a fourdimensional infinite system to find the precise winding numbers). One could conjecture that as we probe loops of higher winding numbers we should eventually find loops with even smaller ratio of total length to the winding number, but in fact it turns out that even loops of winding numbers of order 50 utilize the loops described in Table I, so it appears that those loops are minimal. It seems likely that the torus is twisted in such a way that it is impossible to redefine the elementary cell so as to make loops in the basic directions always an optimal choice as components for loops of higher winding numbers. 

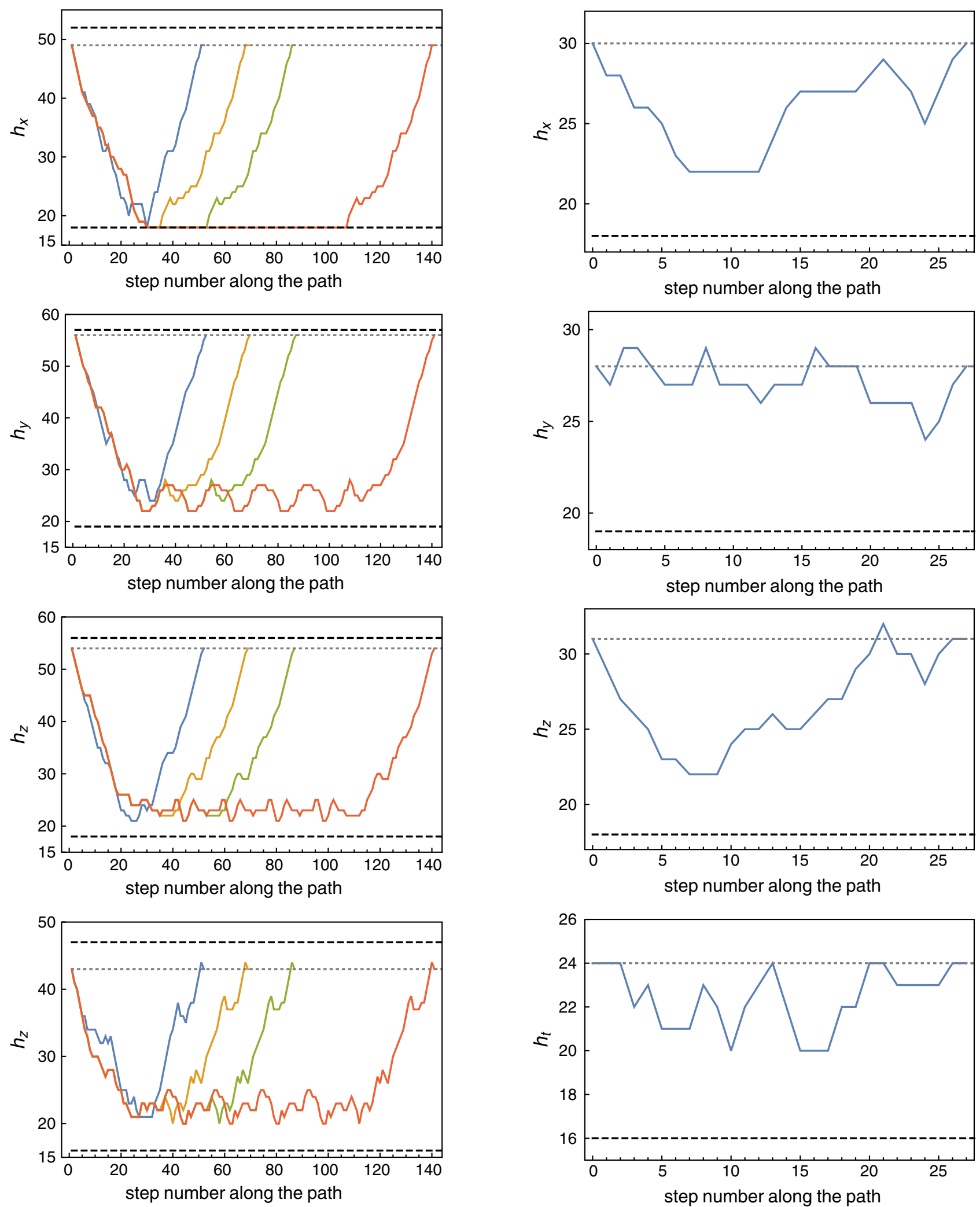

FIG. 7. Heights in the 4 basic directions of consecutive simplices along loops of winding numbers $\{1,0,0,0\}$ (blue), $\{2,0,0,0\}$ (orange), $\{3,0,0,0\}$ (green), $\{6,0,0,0\}$ (red) starting from a simplex in an outgrowth. The dashed lines indicate the minimal and maximal heights in the configuration, and the dotted line indicates the height of the initial simplex.

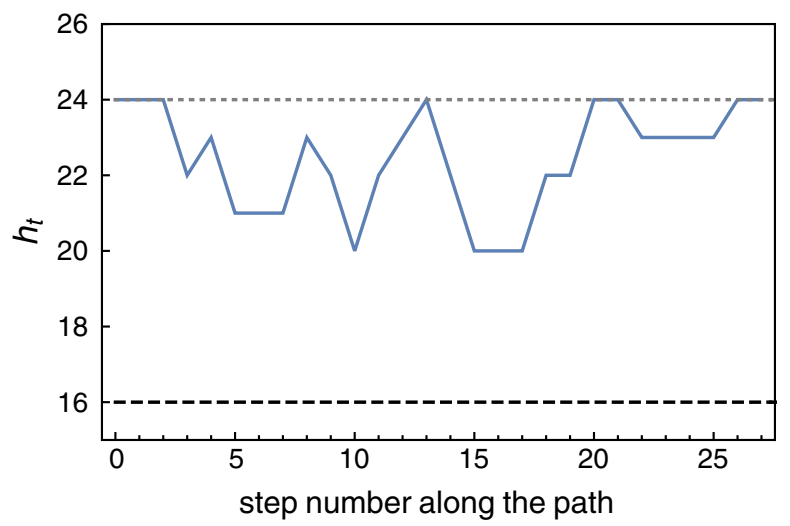

FIG. 8. The four basic heights of simplices belonging to one of the loops from Table I: a loop of length 27 with winding numbers $\{2,-1,1,0\}$. 

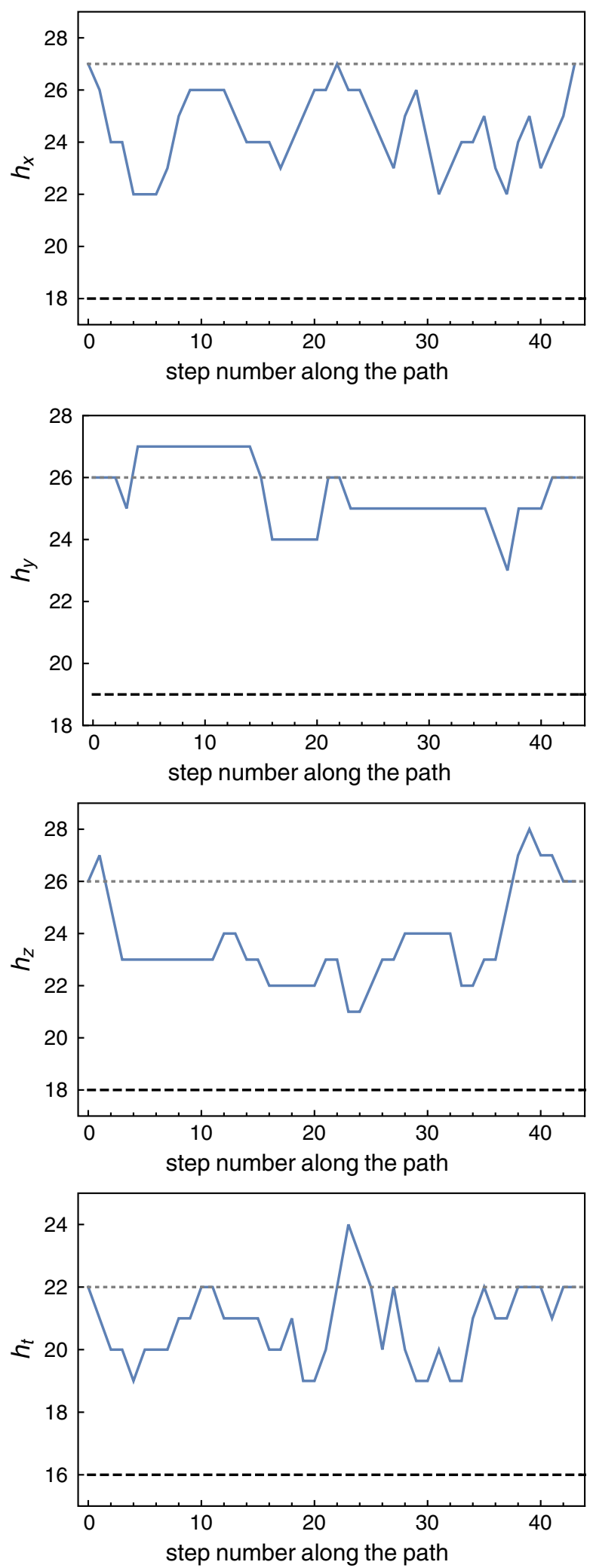

FIG. 9. The four basic heights of simplices belonging to one of the loops from Table I: a loop of length 43 with winding numbers $\{-2,3,-1,1\}$.
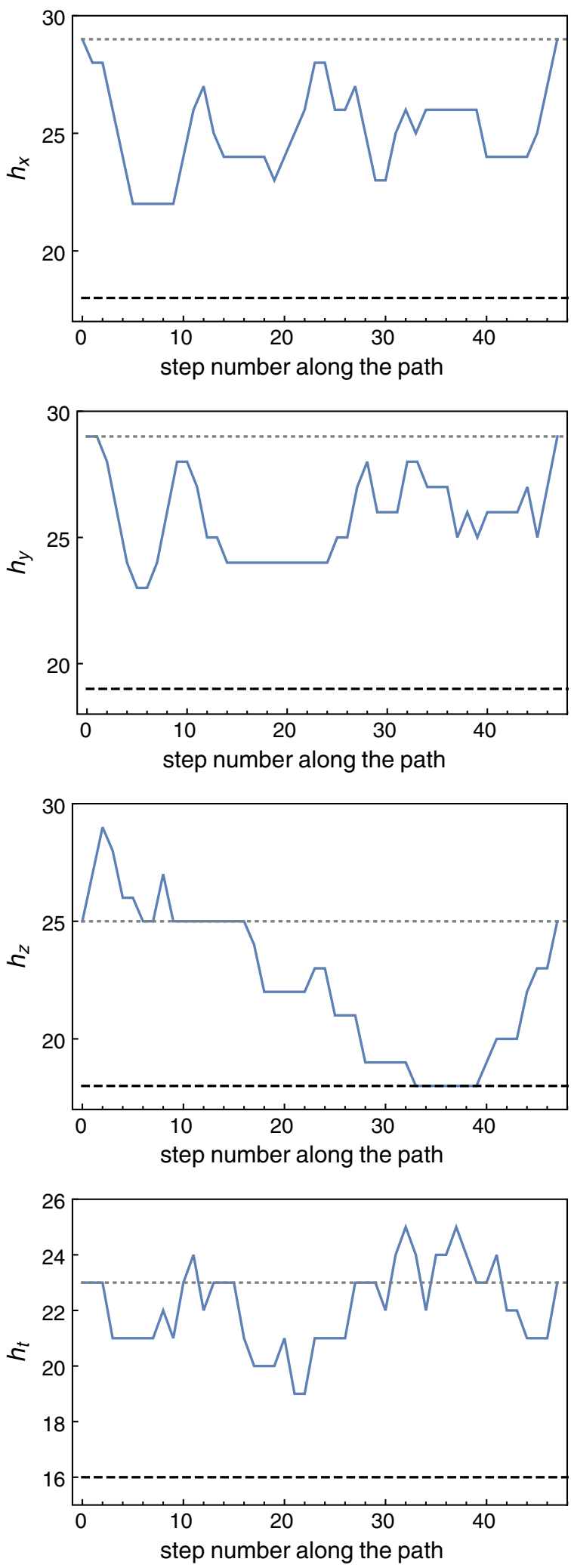

FIG. 10. The four basic heights of simplices belonging to one of the loops from Table I: a loop of length 47 with winding numbers $\{2,-1,3,0\}$. 


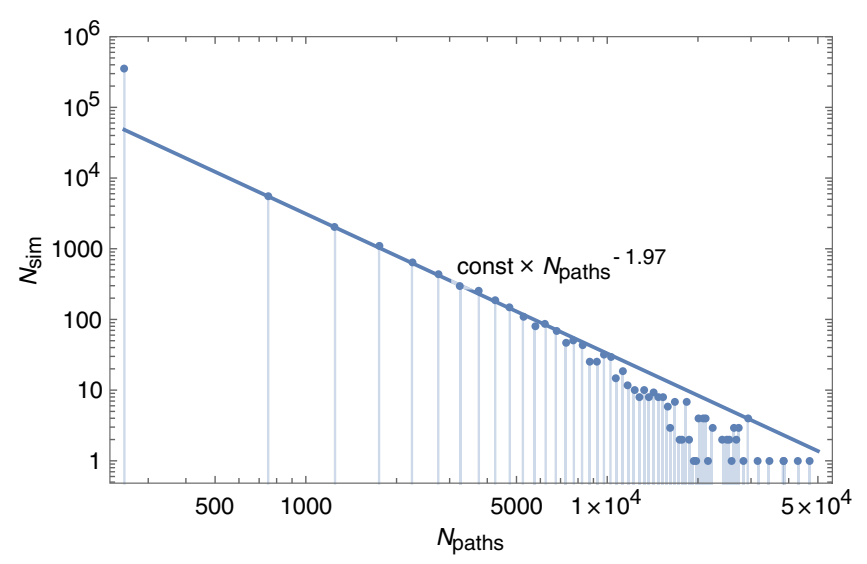

FIG. 11. A log-log scale histogram of the number of simplices crossed by a given number of shortest loops.

Figures $8-10$ show the heights in the basic directions of the consecutive simplices along the shortest loops described above. We can see that the heights, while not minimal, are quite low compared to the average (which is, as mentioned before, above 30). This signifies that these paths too belong to the base ("bulk") region of the torus and are not composed of generic simplices, which mostly belong to the fractal structures.

The algorithm we used creates a diffusion wave starting from a chosen simplex. For each simplex reached in consecutive steps it stores one of the simplices from which it came. In this way, we obtain at the same time not only the lengths of loops of winding number $n$ in the chosen direction but also the lists of simplices along those loops. For each simplex in the configuration, we found and wrote down one shortest path connecting it with its nearest copy in the $\mathrm{x}$-direction. In this way we obtained the lists of simplices belonging to 370724 shortest loops. We repeated the same process in the $y$ - and z-directions. Next, we removed from each list the initial and final simplex of each loop, and we counted the number of appearances of each simplex in the lists. A log-log scale histogram is shown in Fig. 11. The maximum value was more than 40000 , which corresponded to one of the bulk simplices, and the minimal value was zero, which occurred numerous times and corresponded to simplices at the far ends of the outgrowths. The latter simplices are not a part of any geodesics apart from those that start within them. We were able to fit to the histogram a power law curve with the exponent very close to -2 , which seems to bear a certain significance. This functional relationship is different than in the cases of, e.g., a branched polymer or a regular lattice. We have not yet found an algorithmic method of constructing a toroidal graph with behavior described by the same exponent. We plan to investigate this point in a future article.

We also noted that the heights of the simplices and the number of shortest loops passing through them are strongly correlated. We sorted the simplices in the order

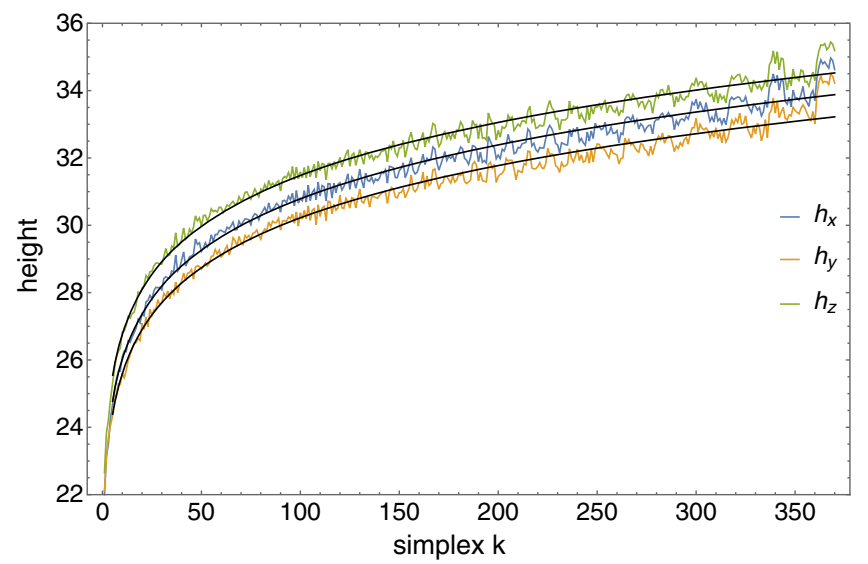

FIG. 12. The $x^{-}, y^{-}$, and $z$-heights of simplices. The simplices were sorted in the order of descending number of loops passing through them, and then the heights were averaged over blocks containing 1000 simplices each. The fitted functions are $h_{x}=21.98 k^{0.0732}, h_{y}=21.66 k^{0.0723}$ and $h_{z}=22.74 k^{0.0706}$.

of descending number of loops passing through them, divided the list into blocks containing 1000 simplices each, and within each block averaged the heights in each of the three spatial directions. With this ordering of simplices, the heights turned out to be increasing functions of the ordinal number of simplices in the list, modulo statistical fluctuations (see Fig. 12). The fluctuations in all three directions were strongly correlated. We fitted a power law to the curves. The exponent is probably the same for all the directions, and the constant factor depends on the shape of the torus-it is higher for the directions in which the torus is more elongated (and so the average height of the simplices is greater).

This shows that the number of loops passing through a given simplex can serve as another indicator of its position within the torus. Most of the geodesics between distant points pass through the bulk simplices in the semiclassical region and do not enter the outgrowths, which are the regions of quantum fluctuations. If a geodesic passes through a simplex in the outgrowth, it usually means that it had its beginning even deeper in the same outgrowth.

\section{NEIGHBORHOODS OF BULK- AND OUTGROWTH-SIMPLICES}

As observed, geodesics between distant simplices tend to pass through simplices of low heights and avoid simplices of middle and greater heights. It is tempting to interpret the former as belonging to a semiclassical "bulk", and the latter to "outgrowths", which are a result of quantum fluctuation. If so, then those regions should differ also in other properties apart from such nonlocal ones as height and the number of loops passing through the simplices. This is in fact the case. The neighborhoods of bulk simplices and 


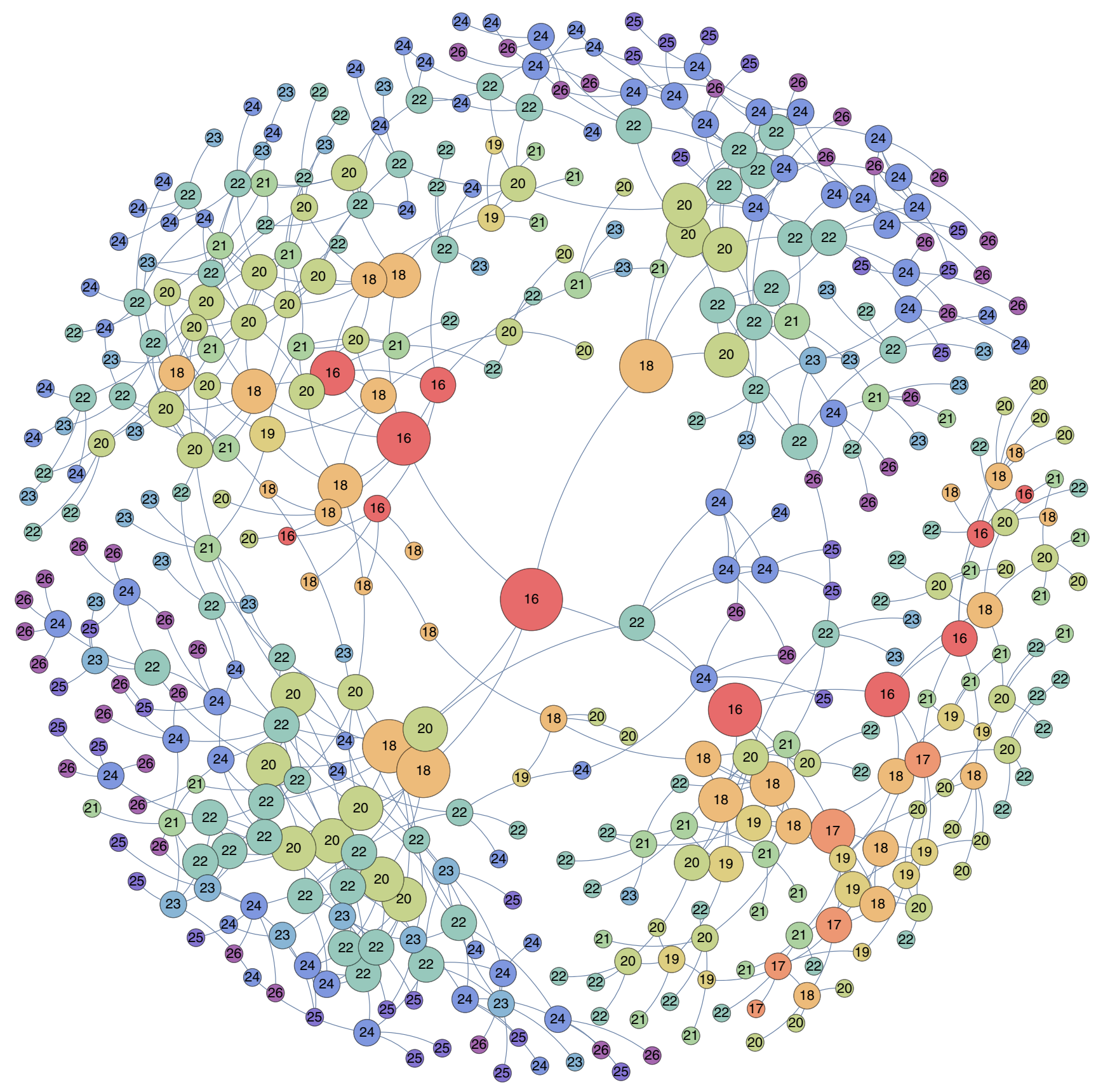

FIG. 13. Six concentric shells around a simplex of height equal to 16. The colors and the numbers indicate the height of a simplex, and the size of a vertex its distance from the starting simplex. The central simplex lies in the bulk region.

outgrowth simplices look considerably different, which allows for a construction of local quantities distinguishing between them.

Figures 13, 14 and 15 show subgraphs of the dual lattice, depicting all the simplices of distance up to 6 from a starting simplex, together with the connections between them. The simplices-vertices of the dual lattice-are represented by circles whose size is a decreasing function of distance from the starting simplex. Colors of the circles indicate heights, with red corresponding to the shortest and violet to the longest loops in a given figure. The heights are also noted as numbers in the circles. ${ }^{3}$

\footnotetext{
${ }^{3}$ Figures in this section are based on data obtained using the version of algorithm with periodic boundary conditions in directions $\mathrm{y}, \mathrm{z}$ and $\mathrm{t}$. Heights defined in this alternative way have similar values and interpretation to $\mathrm{x}$-heights defined previously, though they are not directly equivalent, e.g., here the minimal value is 16 , which occurs in simplices that form the shortest loop with winding numbers $\{1,-1,1,0\}$ (see Table I).
} 


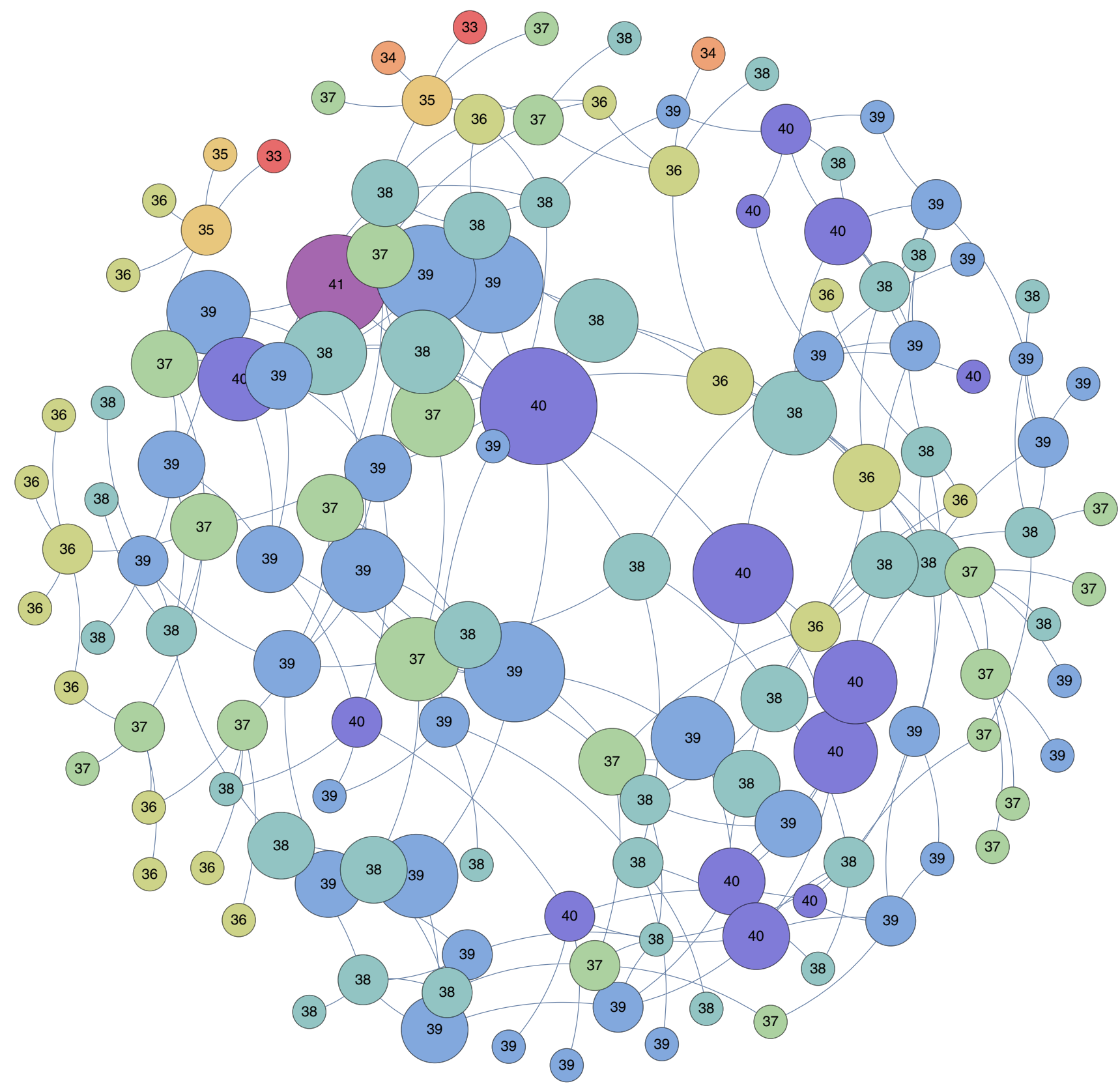

FIG. 14. Six concentric shells around a simplex of height equal to 40. The colors and the numbers indicate the height of a simplex, and the size of a vertex its distance from the starting simplex. The central simplex lies near the deep end of an outgrowth.

In Fig. 13 the starting simplex had height equal to 16. As that is the minimal value, we can see that in the neighborhood of the simplex height tends gradually to increase together with distance from it. By the same token, loop length decreases with increasing distance from a simplex with height equal to 40 , which is among largest in the configuration-Fig. 14. Even a cursory glance at the graphs, moreover, suffices to note a strong dependence of the total number of simplices of distance up to 6 from the starting simplex on its loop-length. As expected, "outgrowth" regions have an elongated shape and a lower
Hausdorff dimension than the "bulk" region, which is a sign of their fractality.

Figure 16 shows the shortest $\{1,-1,1,0\}$ loop and its neighborhood. Comparing it also with Table I, we note that it is among the very shortest nontrivial loops in the configuration. It is the only loop with that set of winding numbers and length 16 . It is readily seen that as we count loops with the same winding numbers and length 17,18 , etc. in the vicinity of the marked loop, the number grows approximately exponentially. 


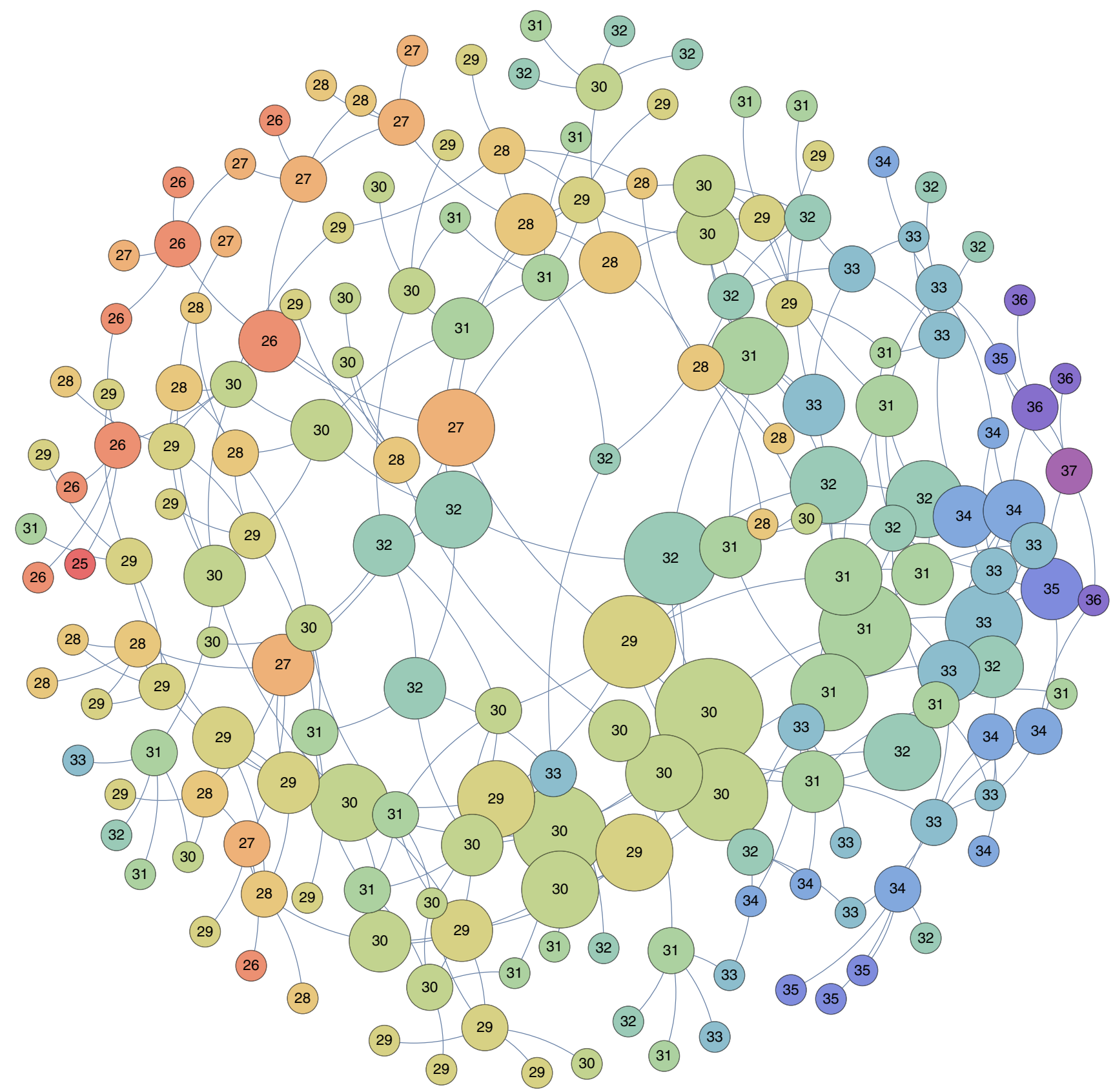

FIG. 15. Six concentric shells around a simplex of height equal to 30. The colors and the numbers indicate the height of a simplex, and the size of a vertex its distance from the starting simplex. The central simplex lies in the middle of an outgrowth.

\section{CONCLUSION}

The detailed measurements performed on a typical toroidal configuration which appears in the CDT path integral in the $C$ phase shows the following: the spatial $T^{3}$ part consists of a relatively small bulk region, the toroidal center, which we have denoted semiclassical, and numerous fractal outgrowths of almost spherical topology (with a single small boundary) which contains most of the simplices. A lower-dimensional illustration of this is shown in the upper part of Fig. 1. Introducing the lengths of the shortest noncontractible loops in the coordinate directions as the heights associate with a given simplex allowed us to classify the simplex as belonging to the toroidal center or to an outgrowth. Further, the number of simplices in the outgrowths where the height is a local maximum is not small. The interpretation of this is that the outgrowths are quite fractal, again in the way illustrated in the upper part of Fig. 1. An important feature of the length distributions of noncontractible loops associated to the simplices is that they scale as $N^{1 / 4}$, where $N$ denotes the size of the 


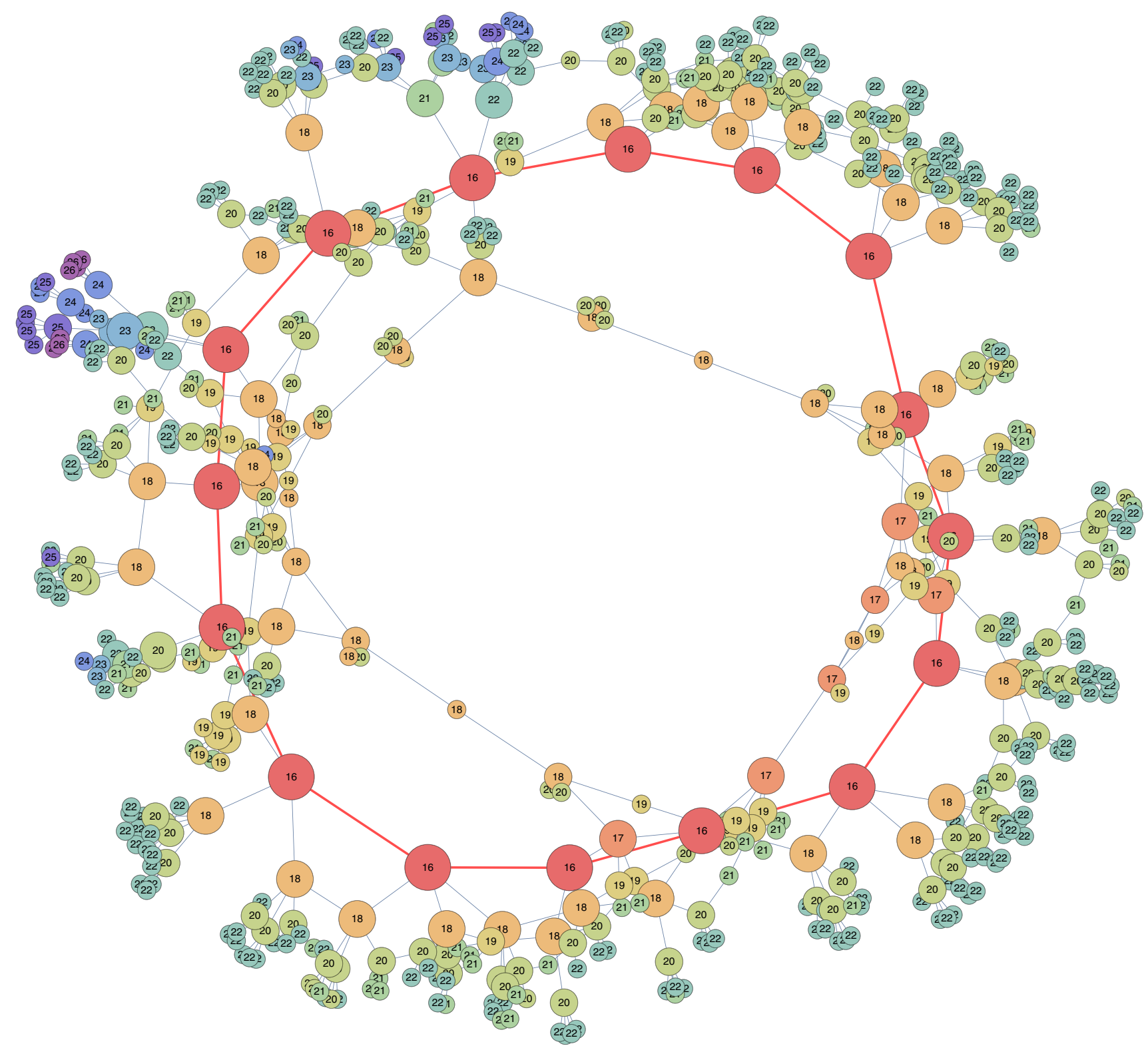

FIG. 16. The shortest $\{1,-1,1,0\}$ loop together with its neighborhood. The colors and the numbers indicate the height of a simplex, and the size of a vertex its distance from the loop.

triangulation, i.e., the number of four-simplices, as shown in Fig. 2. The most likely consequence of such a "canonical" scaling is that the volume of the toroidal center, although small compared to the volume of the outgrowths, will also scale with $N$. It might thus be justified to think of it as semiclassical, in contrast to the toroidal center-part of the two-dimensional configuration shown in the lower part of Fig. 1, which vanishes in the large $N$ limit, as discussed in the Introduction.

We conclude that there is a well defined geometric structure underlying the typical path integral configuration of $T^{4}$ in CDT. It is somewhat more fractal than we had hoped for in the sense that the outgrowths contain most of the simplices, but it invites to use a classical scalar field to define a coordinate system, a procedure common in classical general relativity. By imposing suitable boundary conditions on the scalar field one can make its values record the structure of the toroidal center well, whereas the field is almost constant in an outgrowth. It thus emphasizes what we have denoted the semiclassical part of the configuration and might be a good choice of coordinates if one wants to construct a semiclassical action. Work in this direction will be reported elsewhere.

\section{ACKNOWLEDGMENTS}

The authors thank Jakub Gizbert-Studnicki and Daniel Németh for many fruitful discussions. J. A. acknowledges 
support from the Danish Research Council Grant Quantum Geometry, Grant No. 7014-00066B.Z. D. acknowledges support from the National Science Centre, Poland, Grant No. 2019/32/T/ST2/00390. A. G. acknowledges support by the National Science Centre, Poland, under Grant No. 2015/17/D/ST2/03479. J. J. acknowledges support from the National Science Centre, Poland, Grant No. 2019/33/B/ST2/00589.
[1] J. Ambjørn, A. Görlich, J. Jurkiewicz, and R. Loll, Nonperturbative quantum gravity, Phys. Rep. 519, 127 (2012).

[2] R. Loll, Quantum gravity from causal dynamical triangulations: A review, Classical Quantum Gravity 37, 013002 (2020).

[3] S. Weinberg, Ultraviolet divergences in quantum theories of gravitation, in General Relativity: An Einstein Centenary Survey (Cambridge University Press, Cambridge, England, 1980), pp. 790-831.

[4] M. Reuter, Nonperturbative evolution equation for quantum gravity, Phys. Rev. D 57, 971 (1998).

[5] A. Codello, R. Percacci, and C. Rahmede, Investigating the ultraviolet properties of gravity with a Wilsonian renormalization group equation, Ann. Phys. (Amsterdam) 324, 414 (2009).

[6] M. Reuter and F. Saueressig, Functional renormalization group equations, asymptotic safety, and quantum Einstein gravity, arXiv:0708.1317.

[7] M. Niedermaier and M. Reuter, The asymptotic safety scenario in quantum gravity, Living Rev. Relativity 9, 5 (2006).

[8] D. F. Litim, Fixed Points of Quantum Gravity, Phys. Rev. Lett. 92, 201301 (2004).

[9] J. Ambjørn and J. Jurkiewicz, Four-dimensional simplicial quantum gravity, Phys. Lett. B 278, 42 (1992).

[10] M. E. Agishtein and A. A. Migdal, Simulations of fourdimensional simplicial quantum gravity, Mod. Phys. Lett. A 07, 1039 (1992).

[11] M. Agishtein and A. Migdal, Critical behavior of dynamically triangulated quantum gravity in four dimensions, Nucl. Phys. B385, 395 (1992).

[12] J. Ambjorn, L. Glaser, A. Goerlich, and J. Jurkiewicz, Euclidian $4 \mathrm{~d}$ quantum gravity with a non-trivial measure term, J. High Energy Phys. 10 (2013) 100.

[13] D. Coumbe and J. Laiho, Exploring euclidean dynamical triangulations with a non-trivial measure term, J. High Energy Phys. 04 (2015) 028.

[14] J. Laiho, S. Bassler, D. Coumbe, D. Du, and J. T. Neelakanta, Lattice quantum gravity and asymptotic safety, Phys. Rev. D 96, 064015 (2017).

[15] J. Ambjørn, J. Jurkiewicz, and R. Loll, Dynamically triangulating Lorentzian quantum gravity, Nucl. Phys. B610, 347 (2001).

[16] J. Ambjørn, J. Jurkiewicz, and R. Loll, Reconstructing the Universe, Phys. Rev. D 72, 064014 (2005).
[17] J. Ambjørn, J. Jurkiewicz, and R. Loll, Emergence of a 4D World from Causal Quantum Gravity, Phys. Rev. Lett. 93, 131301 (2004).

[18] J. Ambjørn, A. Görlich, J. Jurkiewicz, and R. Loll, Nonperturbative quantum de Sitter universe, Phys. Rev. D 78, 063544 (2008).

[19] J. Ambjørn, A. Görlich, J. Jurkiewicz, and R. Loll, Planckian Birth of a Quantum de Sitter Universe, Phys. Rev. Lett. 100, 091304 (2008).

[20] J. Ambjørn, Z. Drogosz, J. Gizbert-Studnicki, A. Görlich, and J. Jurkiewicz, Pseudo-Cartesian coordinates in a model of causal dynamical triangulations, Nucl. Phys. B943, 114626 (2019).

[21] J. Ambjørn, J. Barkley, and T. Budd, Roaming moduli space using dynamical triangulations, Nucl. Phys. B858, 267 (2012).

[22] H. Kawai, N. Kawamoto, T. Mogami, and Y. Watabiki, Transfer matrix formalism for two-dimensional quantum gravity and fractal structures of space-time, Phys. Lett. B 306, 19 (1993).

[23] J. Ambjørn and Y. Watabiki, Scaling in quantum gravity, Nucl. Phys. B445, 129 (1995).

[24] J. Ambjørn, J. Jurkiewicz, and Y. Watabiki, On the fractal structure of two-dimensional quantum gravity, Nucl. Phys. B454, 313 (1995).

[25] J. Ambjorn and R. Loll, Nonperturbative Lorentzian quantum gravity, causality and topology change, Nucl. Phys. B536, 407 (1998).

[26] T. Regge, General relativity without coordinates, Nuovo Cimento 19, 558 (1961).

[27] J. Ambjorn, J. Jurkiewicz, and R. Loll, Dynamically triangulating Lorentzian quantum gravity, Nucl. Phys. B610, 347 (2001).

[28] J. Ambjørn, J. Gizbert-Studnicki, A. Görlich, J. Jurkiewicz, and D. Németh, Towards an UV fixed point in CDT gravity, J. High Energy Phys. 07 (2019) 166.

[29] J. Ambjørn and J. Jurkiewicz, On the exponential bound in four dimensional simplical gravity, Phys. Lett. B 335, 355 (1994).

[30] J. Ambjørn, J. Gizbert-Studnicki, A. Görlich, and J. Jurkiewicz, The effective action in 4-dim CDT. The transfer matrix approach, J. High Energy Phys. 06 (2014) 034.

[31] J. B. Hartle and S. W. Hawking, Wave function of the Universe, Phys. Rev. D 28, 2960 (1983).

[32] P. Horava, Quantum gravity at a Lifshitz point, Phys. Rev. D 79, 084008 (2009). 
[33] P. Horava and C. M. Melby-Thompson, General covariance in quantum gravity at a Lifshitz point, Phys. Rev. D 82, 064027 (2010).

[34] C. Anderson, S. J. Carlip, J. H. Cooperman, P. Horava, R. K. Kommu, and P. R. Zulkowski, Quantizing Horava-Lifshitz gravity via causal dynamical triangulations, Phys. Rev. D 85, 044027 (2012).
[35] J. Ambjorn, A. Gorlich, S. Jordan, J. Jurkiewicz, and R. Loll, CDT meets Horava-Lifshitz gravity, Phys. Lett. B 690, 413 (2010).

[36] J. Ambjørn, L. Glaser, Y. Sato, and Y. Watabiki, 2d CDT is 2d Hořava-Lifshitz quantum gravity, Phys. Lett. B 722, 172 (2013). 